\title{
IdeAs
}

Idées d'Amériques

$17 \mid 2021$

Villes et culture dans les Amériques

\section{Irréparable : Résistance et politiques de respectabilité en Colombie}

\section{Anthony Dest}

Traducteur : Laura Cahier

\section{OpenEdition}

Journals

Édition électronique

URL : https://journals.openedition.org/ideas/11179

DOI : 10.4000/ideas. 11179

ISSN : 1950-5701

Éditeur

Institut des Amériques

\section{Référence électronique}

Anthony Dest, «Irréparable : Résistance et politiques de respectabilité en Colombie », IdeAs [En ligne], 17 | 2021, mis en ligne le 01 mars 2021, consulté le 03 juin 2021. URL : http:// journals.openedition.org/ideas/11179; DOI : https://doi.org/10.4000/ideas.11179

Ce document a été généré automatiquement le 3 juin 2021.

\section{(c) ()) (9)}

IdeAs - Idées d'Amériques est mis à disposition selon les termes de la licence Creative Commons Attribution - Pas d'Utilisation Commerciale - Pas de Modification 4.0 International. 


\title{
Irréparable : Résistance et politiques de respectabilité en Colombie
}

\author{
Anthony Dest \\ Traduction : Laura Cahier
}

Remerciements

J'ai grandement apprécié les conversations approfondies que j'ai eues autour de cet article avec Daniel Campo, Sofía Garzón, Óscar Pedraza, Luciane Rocha, Gimena Sánchez-Garzoli, Shreyas Sreenath, Elizabeth Velásquez Estrada et Coletta Youngers, ainsi que les commentaires de Laura Cahier et Laetitia Braconnier-Moreno.

In every era the attempt must be made anew to wrest tradition away from a conformism that is about to overpower it. ${ }^{1}$

\section{Rumeurs et Hummers}

1 Dans une salle de conférence située à quelques rues de la Maison Blanche, une fonctionnaire de l'ambassade de Colombie déclara que la victime défendait les trafiquants de drogue et était connu pour conduire dans un Hummer autour de Cali ${ }^{2}$. La personne en question - Hernan Darío Escobar, un avocat colombien - avait été visée par une fusillade en voiture en mai 2011. La fonctionnaire de l'ambassade laissait ainsi entendre qu'Hernán Darío Escobar n'était pas un "noble leader social», ni un «champion des opprimés » - selon elle, il avait eu ce qu'il méritait. Tandis qu'Escobar était mourant dans un hôpital, elle et d'autres fonctionnaires du gouvernement colombien essayaient d'éviter un fiasco public en diffusant un tel récit parmi leurs nombreux contacts au Congrès américain. Depuis des années, ils tentaient de faire adopter l'accord de libre-échange (ALE) entre les États-Unis et la Colombie, et l'assassinat d'un autre leader social menaçait soudainement de ruiner leurs plans.

2 Au sein de l'organisation de défense des droits humains pour laquelle je travaillais à l'époque, nous nous empressâmes alors de chercher des faits concernant le meurtre et de les replacer dans un contexte de violence généralisée contre les syndicats et les 
militants des mouvements sociaux en Colombie. Les rumeurs émanant de l'ambassade colombienne étaient destinées à contredire notre communiqué de presse initial sur la tentative d'assassinat. En tant qu'activistes dévoués, nous connaissions les efforts entrepris par le gouvernement pour minimiser les assassinats de leaders sociaux, notamment en souillant leurs noms. De notre côté, en présentant publiquement les événements comme une atteinte aux droits des coupeurs de canne à sucre qui étaient représentés bénévolement par Escobar, nous fîmes valoir que cet acte de violence voulait dire quelque chose.

3 Cela fait près d'une décennie que cette expérience me trouble. Évidemment, l'attitude de la fonctionnaire de l'ambassade concernant le meurtre d'un être humain était de mauvais goût, mais elle n'avait rien de surprenant. Alors que nous dénoncions les meurtres et menaces de mort à l'encontre des leaders sociaux, l'ambassade colombienne appelait à la patience et au respect des procédures : les autorités semblaient toujours «savoir » quand un bienfaiteur avait un « côté sombre ». En 2017, l'ancien Ministre de la défense, Luis Carlos Villegas, avait d'ailleurs attribué l'« immense majorité » des meurtres de leaders sociaux à des «disputes relatives aux limites ou demandes de propriété, ou aux luttes liées aux revenus illicites » (El Espectador, 2017). Ce genre de remarques calomnieuses constitue une tentative d'écarter toute idée que les militants pourraient être visés en raison de leur travail politique ou que la violence puisse être associée à la continuité du conflit armé. L'attitude hostile de l'État à l'égard des militants garantit finalement des enquêtes sans issue et, dans la plupart des cas de violence, l'impunité.

4 Mon problème portait cependant sur la façon dont j'interagissais avec la définition même de la violence apportée par l'État. Au lieu de rejeter la violence et son utilisation comme moyen de légitimation du pouvoir de l'État, je soutenais la politique de respectabilité implicitement présente dans les formes libérales d'activisme lié aux droits humains. Les féministes noires ont d'ailleurs longtemps critiqué les limites de la politique de respectabilité. Au lieu de rechercher la reconnaissance en présentant un citoyen modèle et irréprochable au nom de la politique de respectabilité, Cohen suggère plutôt d'adopter une " politique de la déviance ». Cette dernière pourrait avoir un " potentiel de transformation plus important [...] pour améliorer radicalement la vie et les possibilités des personnes les plus vulnérables dans les communautés noiresc ${ }^{3}$ (Cohen C., $2004: 30$ ). Cependant, afin que la mort d'Escobar eût de l'importance aux yeux des décideurs politiques aux États-Unis et en Colombie, sa réputation devait être intacte. L'adhésion à une politique de respectabilité garantit en effet que certaines vies soient défendues, tandis que d'autres demeurent indéfendables. Dans ce cas, elle permettait à l'État de contrôler la conversation.

\section{Dissuader les résistances}

Ce type de dénigrement s'appuie sur le pouvoir de la politique de respectabilité pour dissuader la résistance. Elles présentent le « criminel » comme un sujet irréparable, et les organisations qui luttent en solidarité avec les supposés "criminels" sont coupables par association. Le conflit armé en Colombie a longtemps servi de prétexte pour stigmatiser les militants. En insinuant simplement qu'un tel ou un tel était lié à un groupe de guérilla, cette personne était alors sujette à de multiples dénominations accablantes : " communiste ", " terroriste ", " narco-terroriste ", "Castro-Chavista ", 
etc. Même les fonctionnaires ayant longtemps participé à la politique électorale ne sont pas à l'abri ; les luttes qui impliquent des formes individuelles de leadership sont très sensibles à ce type de stigmatisation.

Les effets de ces dynamiques sont particulièrement préjudiciables pour les plus vulnérables. Notamment, pour activistes dans les luttes pour les droits et les ressources naturelles, être accusé d'entretenir des liens avec un groupe armé ou des trafiquants de drogue peut avoir des conséquences fatales. Le nombre croissant d'assassinats répertoriés par les organisations de défense des droits humains témoigne de l'ampleur de cette violence. Les accusations peuvent également conduire à des peines de prison. $\mathrm{Au}$ cours des dix dernières années, trois de mes proches collègues du Processus des communautés noires (Proceso de Comunidades Negras, PCN) ont passé plusieurs années en prison, sur la base de fausses allégations concernant leur prétendu soutien à des groupes de guérilla. Une énergie considérable est consacrée à blanchir leur nom, avec pour espoir de construire une solide campagne de solidarité afin de les soutenir, ainsi que leurs familles et leurs communautés. Cependant, pour de nombreuses organisations, lutter contre la stigmatisation d'une éventuelle culpabilité s'est avéré trop lourd ; ils ont ainsi fait le choix de soutenir une autre cause, moins compromettante.

7 À ce titre, les défenseurs des droits humains peuvent devenir des « agents actifs dans la guerre du silence» (Taussig M., $1989: 20$ ). Dans le contexte du conflit colombien, la demande chimérique de cas précis de victimes parfaitement innocentes empêche de s'attaquer aux réalités complexes auxquelles les individus sont confrontés. Dans leur analyse de la manière dont les victimes sont neutralisées en tant que sujets politiques dans l'imaginaire libéral " post-conflit », Pedraza et Álvarez expliquent :

Although in practice the ways in which victims define their experiences are not reducible to homogeneous or static categories, the political and legal experiences of what has become known as the "victim" have generally been reduced to a series of moral elements that vacate the political character of their history and subsume them into a narrative of trauma, suffering, and pain.

(Pedraza Ó. \& Álvarez C., 2016 : 175) ${ }^{4}$

Cette moralité est particulièrement présente dans des régions du pays comme le nord du Cauca, fortement touchées par la violence et les trafics de drogue. Par exemple, après le meurtre d'un autochtone dans une ville productrice de coca en septembre 2020, un militant associé au mouvement autochtone s'est assuré d'indiquer dans sa dénonciation publique que la victime "n'était pas un dirigeant autochtone». Cette dénonciation impliquait que le meurtre aurait pu être plus grave si la victime avait été l'un des leaders autochtones. Il a également fait en sorte que le mouvement puisse éviter les dangers de revendiquer un criminel potentiel comme l'un des leurs. En tant qu'abolitionniste, Gilmore aborde ce "problème de l'innocence» de la manière suivante: "To insist on innocence is to surrender politically because 'innocence' evades a problem abolition is compelled to confront: how to diminish and remedy harm as against finding better forms of punishment $»^{5}$ (Gilmore R., $2017: 236$ ). En tant que tels, ces types de renonciations et les récits de pure victimisation contribuent au discours dominant d'une violence juste/injuste, avec tous ses fondements moraux et punitifs. Selon les termes de Taussig, cette capitulation politique «est plus que la production de silence. C'est faire taire, ce qui est tout à fait différent ${ }^{6}$ (Taussig M., 1989 : 14). 


\section{Au-delà de la politique de respectabilité}

9 La politique de respectabilité réduit au silence. Le meurtre d'Escobar a finalement disparu de notre travail au sein de l'organisation des droits humains. Nous n'avons pas cessé de dénoncer la violence, mais nous nous sommes concentrés sur d'autres affaires qui avaient le potentiel de nous donner un avantage politique indiscutable. Le choix des affaires à traiter se faisait en passant au crible d'innombrables exemples dans le but de trouver des éléments gagnables et univoques, ce qui nous a également amenés à nous pencher sur ce qui détermine la respectabilité et la criminalité. Pendant ce temps, les affaires dont les détails étaient à la limite du «criminel » languissaient souvent en toute impunité. Bien que cette stratégie permette un certain degré de traction avec les institutions, elle a pour effet de rendre beaucoup plus d'affaires «hors limites ». Le rejet de la politique de respectabilité peut rendre difficile, voire impossible, l'accès aux institutions qui sont pourtant centrales pour la défense des droits humains. Cependant, le rejet de la politique de respectabilité a également le potentiel de perturber un système politique fondé sur l'utilisation de la violence contre les sujets qu'il construit comme étant « irrécupérables ».

Bibliographie

Benjamin, Walter, "Theses on a Philosophy of History", in Hannah Arendt (ed.), Illuminations: Essays and Reflections, New York, NY: Schocken Books, 2007, p. 253-264.

Cohen, Cathy, "Deviance as Resistance: A New Research Agenda for the Study of Black Politics", Du Bois Review, vol. 1, 2004, p. 27-45.

13 El Espectador, "Asesinatos de líderes son por 'líos de faldas': ministro de Defensa", El Espectador, 17 December 2017, https://www.elespectador.com/noticias/politica/ asesinatos-de-lideres-son-por-lios-de-faldas-ministro-de-defensa/, consulté le 13 octobre 2020.

Gilmore, Ruth Wilson, "Abolition Geography and the Problem of Innocence", in Gaye Theresa Johnson and Alex Lubin (eds.), Futures of Black Radicalism, London, Verso, 2017, p. 225-240.

15 Pedraza, Óscar et Camilo Álvarez, "Maquinarias transicionales y neutralización política de las víctimas en Colombia", inNeyla Graciela Pardo Abril and Juan Ruiz Celis, Víctimas, memoria y justiciar: aproximaciones latinoamericanas al caso colombiano, Bogotá, Colombia, Universidad Nacional de Colombia, 2016.

Taussig, Mick, “Terror as Usual: Walter Benjamin's Theory of History as a State of Siege", Social Text, n²3, 1989, p. 3-20.

\section{NOTES}

1. Traduction: "A chaque époque, il convient d'essayer à nouveau d'arracher la tradition à un conformisme qui est sur le point de la dominer." Traduction de l'anglais au français réalisée par la traductrice. 
2. Cet article est basé sur mon expérience de travail dans une organisation non gouvernementale (ONG) de défense des droits de l'homme à Washington, DC, entre 2010 et 2012. Depuis, j'ai continué à travailler en étroite collaboration avec des militants des droits de l'homme sur diverses questions, en tant qu'universitaire et dans le cadre de mes engagements politiques.

3. Version originale : "transformative potential [...] for radically improving the lives and possibilities of those most vulnerable in Black communities". Traduction de l'anglais vers le français réalisée par la traductrice.

4. "Bien que, dans la pratique, les façons dont les victimes définissent leurs expériences ne soient pas réductibles à des catégories homogènes ou statiques, les expériences politiques et juridiques de celui que l'on appelle la "victime» ont généralement été réduites à une série d'éléments moraux qui libèrent le caractère politique de leur histoire et le submergent dans un récit de traumatisme, de souffrance et de douleur. " Traduction de l'anglais vers le français réalisée par la traductrice.

5. “[...] insister sur l'innocence constitue une capitulation politique, parce que l'innocence échappe à un problème auquel l'abolition est obligée de faire face : comment réduire et réparer le préjudice, plutôt que de trouver de meilleures formes de punition." Traduction de l'anglais vers le français réalisée par la traductrice.

6. Version originale : "[...] is more than the production of silence. It is silencing, which is quite different." Traduction de l'anglais vers le français réalisée par la traductrice.

\section{AUTEURS}

\section{ANTHONY DEST}

Anthony Dest est professeur adjoint au Département d'anthropologie du Lehman College, à New York. Sa thèse de doctorat, intitulée "After the War : Violence and Resistance in Colombia" (Université du Texas, Austin), a reçu le prix de meilleure thèse de l'année 2019 par la Peace and Justice Studies Association. Ses recherches ont été soutenues par des bourses de la National Science Foundation, du Social Science Research Council et de l'Inter-American Foundation.

anthony.dest@lehman.cuny.edu 